\title{
Intrahepatic microcirculatory disorder, parenchymal hypoxia and NOX4 upregulation result in zonal differences in hepatocyte apoptosis following lipopolysaccharide- and D-galactosamine-induced acute liver failure in rats
}

\author{
MASATAKE TANAKA $^{1}$, KOSUKE TANAKA $^{1}$, YUKO MASAKI $^{1}$, MASAYUKI MIYAZAKI $^{1}$, MASAKI KATO $^{1}$, \\ KAZUHIRO KOTOH $^{1}$, MUNECHIKA ENJOJI ${ }^{2}$, MAKOTO NAKAMUTA ${ }^{3}$ and RYOICHI TAKAYANAGI ${ }^{1}$ \\ ${ }^{1}$ Department of Medicine and Bioregulatory Science, Graduate School of Medical Sciences, Kyushu University, \\ Fukuoka 812-8582; ${ }^{2}$ Faculty of Pharmaceutical Sciences, Fukuoka University, Fukuoka 814-0180; \\ ${ }^{3}$ Department of Gastroenterology, Kyushu Medical Center, National Hospital Organization, Fukuoka 810-8563, Japan
}

Received November 13, 2013; Accepted November 27, 2013

DOI: $10.3892 /$ ijmm.2013.1573

\begin{abstract}
Although the mechanisms responsible for acute liver failure (ALF) have not yet been fully elucidated, studies have indicated that intrahepatic macrophage activation plays an important role in the pathogenesis of ALF through intrahepatic microcirculatory disorder and consequent parenchymal cell death. Intrahepatic microcirculatory disorder has been demonstrated in animal models using intravital microscopy; however, the limitations of this method include simultaneously evaluating blood flow and the surrounding pathological changes. Therefore, in this study, we devised a novel method involving tetramethylrhodamine isothiocyanate (TRITC)dextran administration for the pathological assessment of hepatic microcirculation. In addition, we aimed to elucidate
\end{abstract}

Correspondence to: Dr Masatake Tanaka, Department of Medicine and Bioregulatory Science, Graduate School of Medical Sciences, Kyushu University, 3-1-1 Maidashi, Higashi-ku, Fukuoka 812-8582, Japan

E-mail: masa-t@med.kyushu-u.ac.jp; salvador1422@yahoo.co.jp

Abbreviations: ALF, acute liver failure; TRITC, tetramethylrhodamine isothiocyanate; LPS, lipopolysaccharide; GalN, D-galactosamine; TNF- $\alpha$, tumor necrosis factor- $\alpha$; NOX, nicotinamide adenine dinucleotide phosphate oxidase; ROS, reactive oxygen species; HIF1- $\alpha$, hypoxiainducible factor 1- $\alpha$; 4-HNE, 4-hydroxy-2-nonenal; HRP, horseradish peroxidase; PBS, phosphate-buffered saline; H\&E, hematoxylin and eosin; DAB, 3,3'-diaminobenzidine tetrahydrochloride; ECL, enhanced chemiluminescence; FFPE, formaldehyde-fixed paraffin-embedded; SEC, sinusoidal endothelial cell

Key words: acute liver failure, macrophage, hypoxia, oxidative stress, microcirculation, apoptosis, nicotinamide adenine dinucleotide phosphate oxidase, pimonidazole, cleaved caspase-3, 4-hydroxy-2nonenal, reactive oxygen species, hypoxia-inducible factor $1-\alpha$ the mechanisms through which intrahepatic microcirculatory disorder progresses with relation to activated macrophages. ALF was induced in Wistar rats by exposure to lipopolysaccharide and D-galactosamine. Intrahepatic microcirculation and microcirculatory disorder in zone 3 (pericentral zone) of the livers of rats with ALF was observed. Immunohistochemical examinations in conjunction with TRITC-dextran images revealed that the macrophages were mainly distributed in zone 2 (intermediate zone), while cleaved caspase-3-positive hepatocytes, pimonidazole and hypoxia-inducible factor 1- $\alpha$ were abundant in zone 3 . We also found that 4-hydroxy2-nonenal and nicotinamide adenine dinucleotide phosphate oxidase (NOX)4-positive cells were predominantly located in the zone 3 parenchyma. The majority of apoptotic hepatocytes in zone 3 were co-localized with NOX4. Our results revealed that the apoptotic cells in zone 3 were a result of hypoxic conditions induced by intrahepatic microcirculatory disorder, and were not induced by activated macrophages. The increased levels of oxidative stress in zone 3 may contribute to the progression of hepatocyte apoptosis.

\section{Introduction}

Acute liver failure (ALF) is a syndrome defined by the sudden onset of severe liver injury, followed by coagulopathy and encephalopathy. The mortality rates for patients with ALF are $50-70 \%$ (1-3). The prognosis of ALF has not improved significantly over the past decades, despite the development of treatments, such as plasma exchange, dialysis and antibiotics. Although liver transplantation is the only promising treatment for ALF, the rapid progression of the disease and the shortage of donors limit the success of this treatment option (3). However, the pathogenesis of ALF is not yet fully understood and therefore prohibits us from establishing new treatments.

It has been suggested that intrahepatic microcirculatory disorder is involved in the pathogenesis of ALF. Intrahepatic macrophage activation has been suggested as an important factor in the pathogenesis of ALF, causing intrahepatic 
microcirculatory disorder, and subsequently, massive levels of hepatocyte death $(4,5)$. Verification that activated macrophages disturb intrahepatic microcirculation has not been achieved due to the difficulties in visualizing the changes occurring in macrophages and microcapillary structure simultaneously.

Intravital microscopy, used for the observation of biological systems with high resolution, is an excellent technique due to its ability to obtain real-time dynamic microcirculatory images $(6,7)$. It has often been used in experimental animal models of liver injury (8-11). The limitations of this method include difficulty in simultaneously evaluating blood flow and surrounding pathological changes. Furthermore, only a limited number of laboratories are able to utilize this technique as it demands expensive and specialized equipment and facilities.

In this study, we designed a method for visualizing hepatic microcapillary structure with the administration of tetramethylrhodamine isothiocyanate (TRITC)-dextran. Applying this method to lipopolysaccharide (LPS)- and D-galactosamine (GalN)-induced liver injury in rats, a widely accepted animal model of ALF (12), we were able to obtain detailed findings of intrahepatic microcirculatory disturbance in relation to intrahepatic macrophage activation. Given that the massive reduction in the number of viable hepatocytes is caused by intrahepatic microcirculatory disorder, there still remains the controversial issue of whether this reduction in ALF is due to apoptosis or necrosis. A number of studies have demonstrated that activated macrophages can cause intrahepatic microcirculatory disorder and consequent parenchymal necrosis (13-18), while others have indicated that activated macrophages induce parenchymal apoptosis by secreting death receptor ligands, such as Fas ligand or tumor necrosis factor- $\alpha$ (TNF- $\alpha)(19,20)$. Although it is difficult to determine which manner of cell death is dominant, recent studies have indicated that apoptosis plays a major role in the reduction in the number of viable hepatocytes during the development of $\operatorname{ALF}(42,43)$.

Apoptosis is induced through the sequential activation of caspases that can be activated by death receptors (extrinsic pathway) also cellular stress (intrinsic pathway) (21). As regards the intrinsic pathway, the nicotinamide adenine dinucleotide phosphate oxidase (NOX) family is a major source of cellular reactive oxygen species (ROS) (22-24). Among NOX homologues, only NOX4 is an inducible isoform (25). To evaluate the mechanisms that trigger hepatocyte apoptosis in ALF, we conducted an immunohistochemical detection of cleaved caspase-3, CD68, pimonidazole, hypoxia-inducible factor 1- $\alpha$ (HIF1- $\alpha$ ), 4-hydroxy-2-nonenal (4-HNE) and NOX4 in rat livers, and aimed to determine correlations between their localization and intrahepatic microcirculatory disorder.

\section{Materials and methods}

Chemicals and antibodies. All reagents were purchased from Sigma Chemical Co. (St. Louis, MO, USA) unless otherwise stated. Monoclonal mouse anti-CD68 and rabbit anti-NOX4 antibodies, as well as horseradish peroxidase(HRP)-conjugated polyclonal goat anti-mouse and goat anti-rabbit antibodies were purchased from Abcam (Cambride, UK). The polyclonal rabbit anti-cleaved caspase-3 and rabbit anti-HIF1- $\alpha$ antibodies were from Novus Biologicals (Littleton, CO, USA). A monoclonal mouse anti-4-HNE antibody was obtained from NOF Corp.
(Tokyo, Japan). Pimonidazole and the mouse anti-pimonidazole adducts monoclonal antibody (Hypoxyprobe ${ }^{\mathrm{TM}}-1 \mathrm{kit}$ ) were purchased from Hypoxyprobe Inc. (Burlington, MA, USA).

Animals. Eight-week-old male Wistar rats (weighing approximately $200 \mathrm{~g}$ ), were purchased from Japan SLC, Inc. (Hamamatsu, Japan). The animals were provided access to food and water ad libitum and maintained on a 12-h light-dark cycle in a temperature $\left(18-21^{\circ} \mathrm{C}\right)$ and humidity $(55 \pm 5 \%)$ controlled environment for 1 week before the experiments. All animals received humane care, and all experiments in this study were performed according to the guidelines of the Committee for the Care and Use of Laboratory Animals at Kyushu University, Fukuoka, Japan.

Experimental protocols. The rats were divided into 2 groups ( $\mathrm{n}=10 \mathrm{rats} / \mathrm{group})$. LPS from Escherichia coli $(5 \mu \mathrm{g} / \mathrm{kg}$ body weight) and GalN (500 $\mathrm{mg} / \mathrm{kg}$ body weight) were intraperitoneally injected into the rats in the ALF group, while sterile saline was injected into the rats in the control group $(\mathrm{n}=10)$. At $22 \mathrm{~h}$ post-administration, pimonidazole $(60 \mathrm{mg} / \mathrm{kg}$ body weight) was intraperitoneally injected into all the rats. Pimonidazole is rapidly reduced in hypoxic cells to a highly reactive intermediate that binds to sulfur on glutathione and proteins. Using an antibody against these pimonidazole-sulfur adducts, immunohistochemistry can be employed to detect the hypoxic cells (26-33). Two hours after the pimonidazole injection, the rats were anesthetized with isoflurane, and the abdomens were incised and canulated at the inferior vena cava using a $20 \mathrm{G}$ plastic needle. After a 1-ml blood sample was withdrawn, the rats were intravenously injected with TRITC-dextran (500 mg/kg body weight) dissolved in $1 \mathrm{ml}$ of sterile saline and the livers excised. One lobe of excised liver was fixed in $20 \%$ formaldehyde solution (Mildform 20N; Wako Pure Chemical Industries, Osaka, Japan) for histological analysis, and another lobe was snap-frozen in liquid nitrogen for use in western blot analysis.

Preparation of liver sections to assess TRITC-dextran distribution. The excised livers were fixed in $20 \%$ formaldehyde solution for 1 day, paraffin-embedded and sectioned $(5 \mu \mathrm{m}$ thickness). The sections were deparaffinized with xylene and rehydrated by washing through a graded alcohol series and deionized water. The hydrated tissue sections were washed with phosphate-buffered saline (PBS). To evaluate TRITCdextran distribution, the sections were mounted in aqueous Fluor Mount/plus (Diagnostic Biosystems Inc., Pleasanton, CA, USA) immediately after deparaffinization and observed under a fluorescence microscope (BZ-9000; Keyence, Osaka, Japan).

Hematoxylin and eosin $(H \& E)$ staining. Some sections were deparaffinized, rehydrated and stained with $H \& E$ to evaluate the general histological state. Some sections were deparaffinized, rehydrated and stained with $\mathrm{H} \& \mathrm{E}$ according to a standard procedure (66) to evaluate the general histological state.

Immunohistochemistry. The liver sections were deparaffinized, rehydrated and exposed to $3 \%(\mathrm{v} / \mathrm{v}) \mathrm{H}_{2} \mathrm{O}_{2}$ for $15 \mathrm{~min}$ at room temperature to quench endogenous peroxidase activity. The sections were incubated with antigen retrieval solution (Target 
Retrieval Solution; Dako, Tokyo, Japan) for $30 \mathrm{~min}$ at $95^{\circ} \mathrm{C}$. Following 2 washes in PBS, the sections were incubated with blocking buffer (Blocking One Histo; Nakalai Tesque, Inc., Kyoto, Japan) for $30 \mathrm{~min}$. The sections were then incubated with antibodies dissolved in Can Get Signal Immunostain solution A (Toyobo, Osaka, Japan) for $1 \mathrm{~h}$ at room temperature, followed by incubation with the appropriate secondary antibody conjugated to HRP in Can Get Signal Immunostain solution B for $30 \mathrm{~min}$ at room temperature. Following 2 washes in PBS, the sections were incubated with 3,3'-diaminobenzidine tetrahydrochloride (DAB) solution for $5 \mathrm{~min}$.

For double immunohistochemical staining, the same steps from antigen retrieval to incubation with a chromogen described above were repeated, except that acetonitrile solution (Vector SG; Vector Laboratories, Burlingame, CA, USA) instead of DAB was used as a chromogen. The sections were washed twice with PBS and mounted in aqueous Fluor Mount/plus. Hematoxylin counterstaining was only conducted for the samples incubated with a single antibody. For CD68 and pimonidazole immunohistochemistry, we obtained bright-field and fluorescent images from the same liver sections and merged them. For double immunohistochemistry and HIF1- $\alpha$, 4-HNE and NOX4 immunohistochemistry, staining was carried out on serial sections. The pathological findings assessed by one of the authors blinded to the group allocations.

Western blot analysis. Liver tissue samples $(20 \mathrm{mg})$ were homogenized in lysis buffer and protein levels were quantified using a commercial protein assay kit (Bio-Rad, Hercules, CA, USA). Liver extracts (30 $\mu \mathrm{g}$ protein/lane) were electrophoresed and subjected to SDS-PAGE under reducing conditions using $10 \%$ polyacrylamide gels. Proteins were transferred to Immobilon-P polyvinylidene difluoride transfer membranes (Millipore, Billerica, MA, USA) and probed with an antiNOX4 rabbit monoclonal antibody. Detection was carried out using an anti-rabbit IgG goat HRP-conjugated antibody in conjunction with ECL Prime Western Blotting Detection reagent (GE Healthcare, UK Ltd., Buckinghamshire, UK). The quantification of enhanced chemiluminescence (ECL) was performed using an ImageQuant LAS 4000 imaging system (GE Healthcare, UK Ltd.).

Statistical analysis. Data are expressed using box and whisker plots. The Steel test was applied to analyze results between 2 groups, and the Steel-Dwass test was used to analyze results between 3 or more groups. A P-value $\leq 0.05$ was considered to indicate a statistically significant difference.

\section{Results}

Histology of livers of rats with LPS/GalN-induced ALF. The liver sections obtained from the control rats had an integrated structure of hepatic lobules (Fig. 1A). The intraperitoneal injection of LPS/GalN induced marked hepatic injuries, accompanied by hemorrhaging and inflammatory cell infiltration (Fig. 1B).

TRITC-dextran distribution in liver sections. We varied the interval from TRITC-dextran injection to liver excision (30 sec, 1, 2, 3, 5 and $10 \mathrm{~min}$ ), and concluded that clear and strong signals of TRITC-dextran could be obtained after 1 min (data not shown). The interval was therefore fixed to $1 \mathrm{~min}$ in subsequent experiments. In the liver sections from the control rats, TRITC-dextran was distributed evenly across the liver acinus (portal veins, sinusoids and central veins) (Fig. 1C and E). In the liver sections from the rats with ALF, TRITC-dextran was clearly distributed in the portal veins and zone 1 (periportal zone) sinusoids, and could be faintly observed in the central veins and zone 3 (pericentral zone) sinusoids. Additionally, several spotty signals corresponding to TRITC-dextran appeared in zone 2 (intermediate zone) (Fig. 1D and F). We measured the ratio of TRITC-dextranpositive areas to the parenchymal area in each zone (Fig. 1G). TRITC-dextran distribution in zone 3 of the livers of rats with ALF was markedly decreased when compared with that in zones 1 and 2 of the livers of rats wtih ALF, and all zones in the livers of the control rats.

Correlation between intrahepatic microcirculation and infiltration of $\mathrm{CD} 68^{+}$positive cells. In the control rats, only some cells were positive for CD68 (Fig. 2C). In the rats with ALF, there was a marked infiltration of $\mathrm{CD} 68^{+}$cells distributed among the liver acinus (Fig. 2D). We observed that $\mathrm{CD}^{2} 8^{+}$cells dominantly localized around the spotty signals of TRITCdextran in zone 2 (Fig. 2F). The ratio of $\mathrm{CD}^{2} 8^{+}$cells to the parenchymal area in each zone indicated that the $\mathrm{CD} 68^{+}$cells in all zones in the livers of rats with ALF were significantly increased in comparison to those in the control rats; the $\mathrm{CD} 68^{+}$ cells were most abundant in zone 2 in the livers of rats with ALF (Fig. 2G).

Apoptotic hepatocytes and their localization. In the control rats, a few cells were positive for cleaved caspase-3 (Fig. 3A). In the rats with ALF, high numbers of cleaved caspase- $3^{+}$ hepatocytes were observed (Fig. 3B) and were mainly localized in zones 2 and 3 (Fig. 3C and D). In the rats with ALF, we evaluated the ratio of cleaved caspase- $3^{+}$hepatocytes to the parenchymal area in each zone (Fig. 3E). Cleaved caspase- $3^{+}$ hepatocytes were most abundant in zone 3 .

Localization of parenchymal hypoxia, HIF1- $\alpha$, oxidative stress and NOX4. On the basis of the results presented above, we hypothesized that hypoxia due to intrahepatic microcirculatory disorder may result in parenchymal oxidative stress and consequent apoptosis in zone 3 via an intrinsic pathway. In the control rats, whole liver tissue was negative for pimonidazole (normoxic conditions) (Fig. 4C). In the rats with ALF, the zone 1 parenchyma was predominantly negative for pimonidazole staining, whereas the zone 3 parenchyma was strongly positive (hypoxic conditions) (Fig. 4D). Hypoxia in zone 3 appeared to be induced by impaired intrahepatic microcirculation (Fig. 4F). An immunohistochemical detection of HIF1- $\alpha$ (Fig. 4G and H), 4-HNE (Fig. 4I and J) and NOX4 (Fig. 4K and L) was conducted. The whole liver tissue from the control rats was mostly negative, whereas the zone 3 parenchyma in the rats with ALF was positive for all proteins. The localization of HIF1- $\alpha, 4-\mathrm{HNE}$ and NOX4 corresponded with that of pimonidazole. NOX4 protein expression was markedly increased in the rats with ALF compared with the control rats (Fig. 4M). 

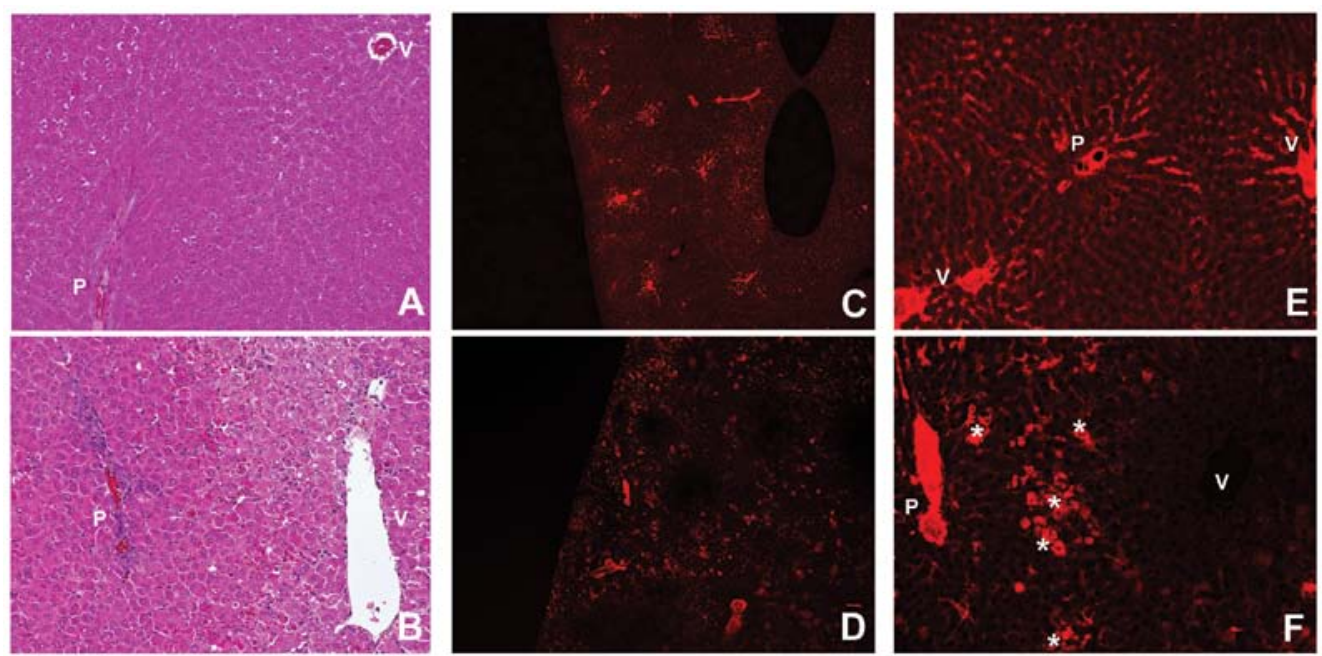

G

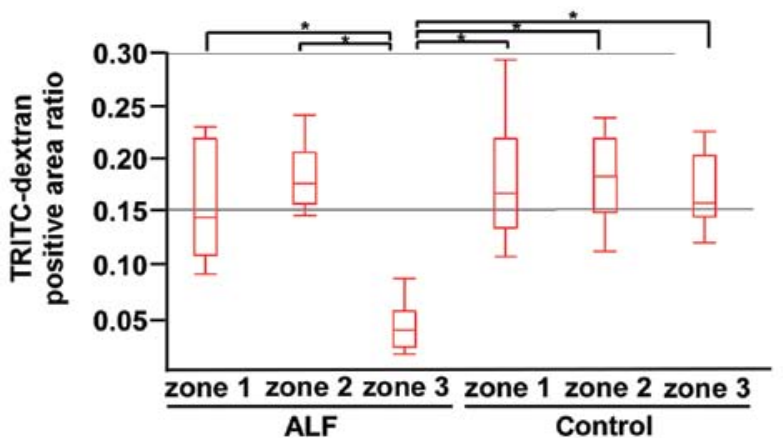

Figure 1. Hematoxylin and eosin (H\&E)-stained sections and fluorescent images from tetramethylrhodamine isothiocyanate (TRITC)-dextran-injected livers. Liver sections from (A) control rats, and (B) rats with acute liver failure (ALF) induced by lipopolysaccharide/D-galactosamine (LPS/GalN) stained with H\&E. Fluorescent images of TRITC-dextran-injected livers from (C and E) the control rats and (D and F) rats with ALF. (G) The TRITC-dextran distribution ratio for each zone in the livers from the control rats and rats with ALF. (C and E) In the livers of the control rats, TRITC-dextran was evenly distributed in the portal veins $(\mathrm{P})$, sinusoids and central veins $(\mathrm{V})$. (D and F) In the livers of rats with ALF, TRITC-dextran was distributed in the portal veins (P) and zone 1 sinusoids, but not in the central veins and zone 3 sinusoids. In zone 2, several spotty signals of TRITC-dextran can be obseved (*). Original magnification, x200 (A, B , E and F), and $x 40$ (C and D). The TRITC-dextran distribution ratio in zone 3 of the livers of rats with ALF was significantly decreased compared with ALF zones 1 and 2, and zones 1-3 in the controls ( $\mathrm{P} \leq 0.05)$. Zone 1, periportal zone; zone 2, intermediate zone; zone 3, pericentral zone.

Analysis of zonal differences in hepatocyte apoptosis. The simultaneous immunohistochemical detection of cleaved caspase-3 and CD68 (Fig. 5A-C) revealed that the majority of the apoptotic hepatocytes in zone 2 were localized adjacent to infiltrated macrophages. Few apoptotic hepatocytes in zone 3 were similarly localized. The simultaneous immunohistochemical detection of cleaved caspase-3 and NOX4 (Fig. 5D-F) revealed that few apoptotic hepatocytes in zone 2 colocalized with NOX4, whereas the majority of apoptotic hepatocytes in zone 3 colocalized with NOX4.

\section{Discussion}

In this study, we demonstrate that the intrahepatic microcirculatory structure can be visualized in formaldehyde-fixed paraffin-embedded (FFPE) liver sections from rats treated with TRITC-dextran. This method can be applied to both healthy and ALF livers stimulated with LPS/GalN. Previously, the administration of fluorescent dextran has been used to analyze tissue microcirculation under various conditions with the aim of demonstrating exceptional blood flow or extravasation. Vollmar et al (34) administered fluorescent dextran intravenously to rats with liver cirrhosis and observed its excretion to the lymph vessels. Sun et al (35) administered fluorescent dextran to rats with ischemia/reperfusion injury via the superior mesenteric artery to visualize extravasation at the intestinal villi. To our knowledge, no preceding study, however, has used this method to assess sinusoidal blood flow. This technically convenient method could be applied to evaluate other animal models mimicking liver diseases, such as graft-versus-host disease, sinusoidal obstruction syndrome and ischemia/reperfusion injury, which encompass intrahepatic microcirculatory disorder during their progression.

In our study, in the livers of rats with ALF, several spotty signals of TRITC-dextran were observed in zone 2 (Fig. 1D and F). These spotty signals seemed to represent a pooling of sinusoidal blood flow as a result of extravasation into the parenchyma. This likely indicates the disrupted integrity of sinusoidal endothelial cells (SECs), which is consistently described in previous studies involving animal models of ALF (36) and patients (37). Additionally, signals of TRITCdextran in zone 3, the downstream region of sinusoidal flow, were markedly reduced (Fig. 1D, F and G). This is a reasonable result due to the destruction of the microcirculatory structure upstream of zone 3. To confirm whether these sinusoidal findings in ALF were connected to hepatic macrophage activation, 

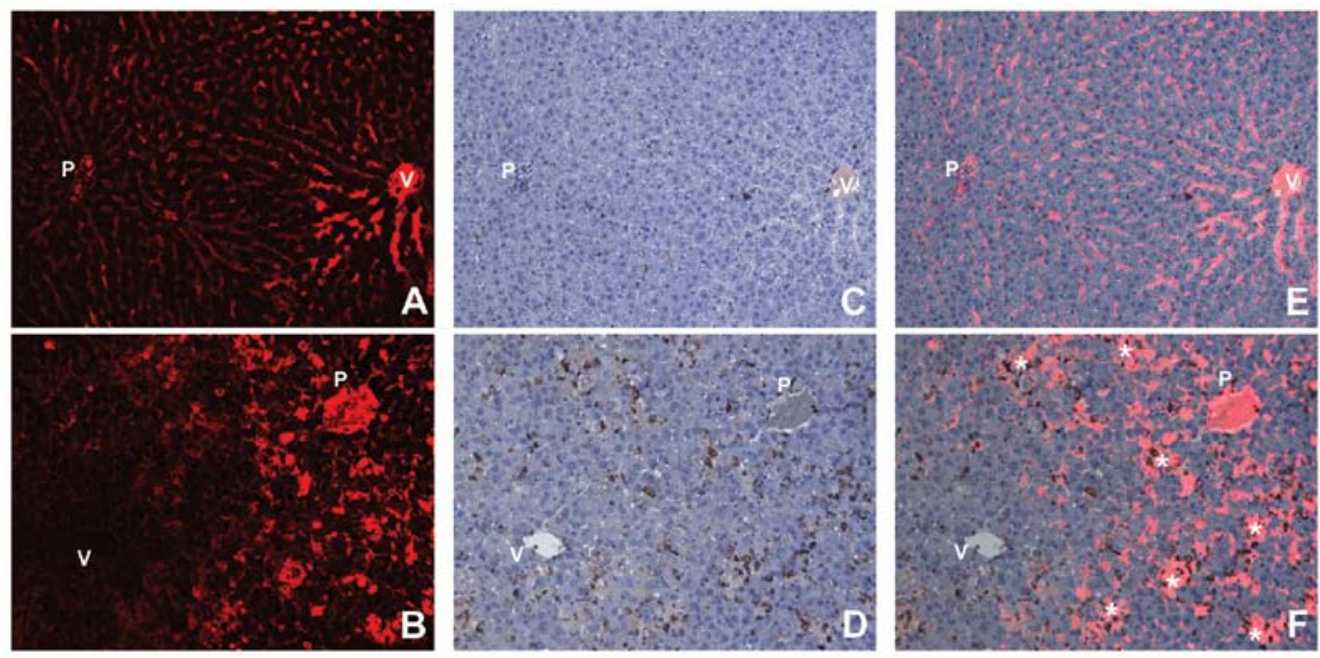

G

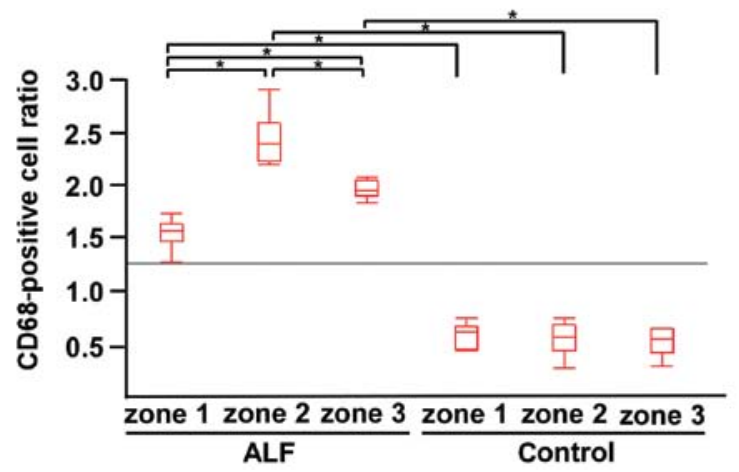

Figure 2. Tetramethylrhodamine isothiocyanate (TRITC)-dextran and CD68 immunohistochemistry. (A and B) TRITC-dextran distribution. (C and D) Immunohistochemical detection of CD68 and (E and F) merge images for controls (upper panel) and acute liver failure (ALF) (lower panel) samples. (G) The $\mathrm{CD}^{+} 8^{+}$cell ratio in each zone for the control and ALF samples. In the controls, TRITC-dextran was evenly distributed in the liver acinus (A) with some CD68 cells (C). In ALF livers, marked infiltration of CD68 ${ }^{+}$was observed in the liver acinus (D). Merge images (B and D) showed that CD68 ${ }^{+}$cells were predominantly localized around the spotty signals of TRITC-dextran in zone $2(*)$ (F) (original magnification, x200). CD68 cells in the ALF samples were significantly increased compared with the controls. (G) In the ALF samples, CD68 ${ }^{+}$cells were most abundant in zone 2, followed by zone 3 and then zone 1 ( $\mathrm{P} \leq 0.05)$. P, portal vein; V, central vein. Zone 1, periportal zone; zone 2, intermediate zone; zone 3, pericentral zone.

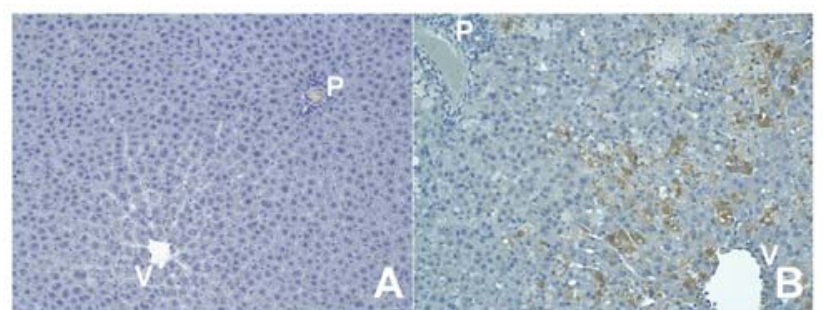

Control

ALF

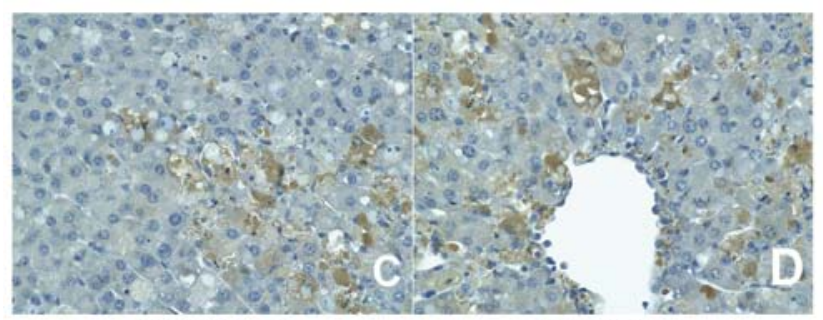

ALF zone 2
ALF zone 3
$\mathbf{E}$

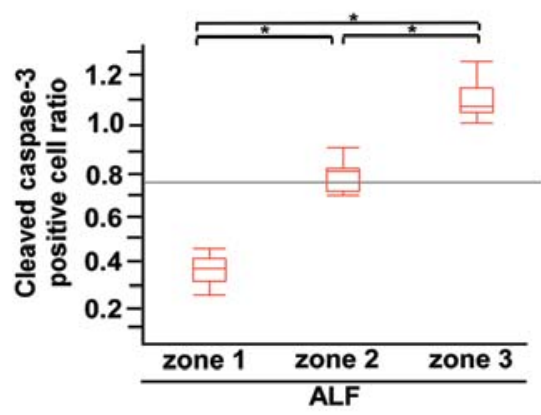

Figure 3. Immunohistochemical detection of cleaved caspase-3 and the apoptotic hepatocyte ratio. Immunohistochemistry for cleaved caspase-3 in livers from (A) control rats and (B-D) rats with acute liver failure (ALF). (E) The cleaved caspase- $3^{+}$cell ratio in each ALF zone. (A) In the controls, few cells were positive for cleaved caspase-3. (B) In the livers of mice with ALF, high numbers of cleaved caspase- $3^{+}$hepatocytes were observed, particularly in zones 2 (C) and 3 (D). Original magnification, x200 (A and B), x400 (C and D). Cleaved caspase- $3^{+}$cell ratios in the livers of rats with ALF were greatest in zone 3, followed by zones 2 and 1 (E) ("P $\leq 0.05)$. P, portal vein; V, central vein. Zone 1, periportal zone; zone 2, intermediate zone; zone 3, pericentral zone. 


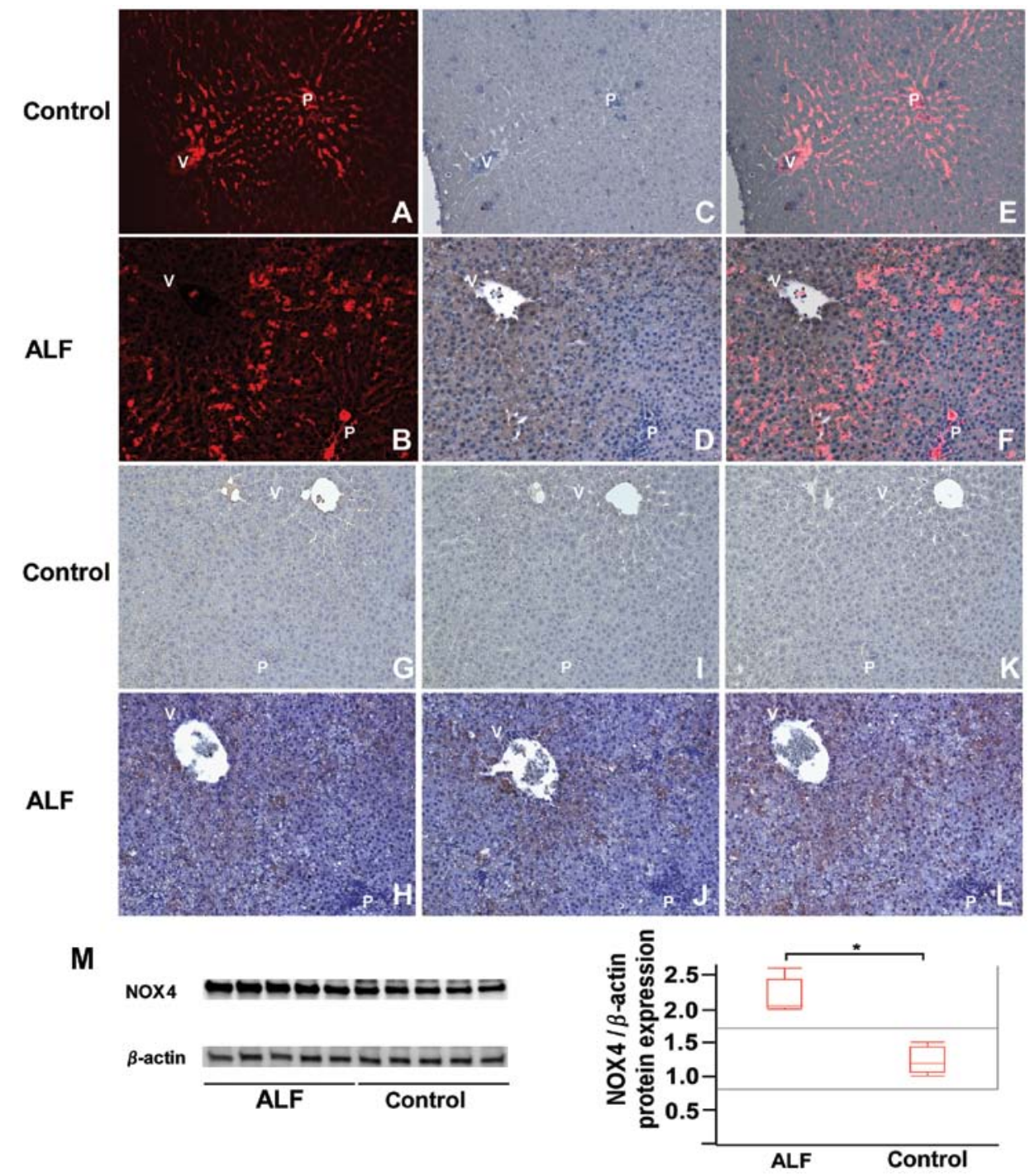

Figure 4. Immunohistochemical detection of pimonidazole, hypoxia inducible factor 1- $\alpha$ (HIF1- $\alpha$ ), 4-hydroxy-2-nonenal (4-HNE) and nicotinamide adenine dinucleotide phosphate oxidase (NOX4), and NOX4 western blot analysis results. (A and B) Tetramethylrhodamine isothiocyanate (TRITC)-dextran distribution. (C and D) Immunohistochemical detection of pimonidazole and (E and F) merge images from the livers of (A, C and E) control rats and (B, D and F) rats with acute liver failure (ALF). Immunohistochemistry for $(\mathrm{G}$ and H) HIF1- $\alpha$, (I and J) 4-HNE and (K and L) NOX4 in livers of (G, I and K) control rats and (H, J and L) rats with ALF. (M) Western blot analysis of NOX4. In the controls, TRITC-dextran was evenly distributed in the liver acinus lacking hypoxic areas (A and C). In the livers of rats with ALF, zone 1 was normoxic with sustained sinusoidal flow, but zone 3 was hypoxic with reduced sinusoidal flow (B, D and F). In the controls, all zones were predominantly negative for HIF1- $\alpha(\mathrm{G})$, 4-HNE (I) and NOX4 (K). In the ALF samples, zone 1 was mainly negative, and zone 3 positive for HIF1- $\alpha(\mathrm{H}), 4-\mathrm{HNE}(\mathrm{J})$ and NOX4 (L) (original magnification, x200). In the livers of rats with ALF, NOX4 protein expression was increased compared with the controls (M) ("P $\leq 0.05)$. P, portal vein; $\mathrm{V}$, central vein. Zone 1, periportal zone; zone 2, intermediate zone; zone 3, pericentral zone.

we generated merged images comprising CD68 immunohistochemistry and TRITC-dextran fluorescent images.

Macrophage activation has been observed in patients with ALF $(19,38)$ and is thought to be an important aspect of the pathogenesis of ALF $(4,5)$. Nonetheless, the pathway from macrophage activation to massive hepatocyte death remains unclear. As sinusoidal fibrin deposition has been observed in patients with ALF $(39,40)$, activated macrophages are thought to induce sinusoidal fibrin deposition and cause hepatic microcirculatory disorder, thereby resulting in parenchymal hypoxia and necrosis (13-18). An essential role of activated macrophages has been reported in parenchymal apoptosis through the secretion of death receptor ligands, such as Fas ligand and TNF- $\alpha(19,20)$. Evidence indicates that the same stimuli can result in either the necrosis or the apoptosis of hepatocytes (21). Bantel et al (41) showed that serum M30, which selectively recognizes a caspase cleaved neoepitope of cytokeratin 18 , reflected hepatocyte apoptosis in patients with chronic hepatitis C. Rutherford et al $(42,43)$ reported the findings of Bantel et al (41) as an effective predictor for the prognosis of ALF. Although the relative contribution of necrosis and apoptosis to ALF remains controversial (44), it is acceptable that a pathway triggered by macrophage activation that results in widespread hepatic hypoxia and subsequent massive hepatocyte death would exist, and that apoptosis would be involved in this process. Therefore, an investigation of the mechanisms of apoptosis in ALF may prove useful in the development of novel treatment strategies to protect liver cells from uncontrollable cell destruction.

In our rat model of $\mathrm{ALF}, \mathrm{CD}^{+} 8^{+}$cells were localized around pooling TRITC-dextran in zone 2 , indicating that infiltrated macrophages directly caused SEC destruction in 


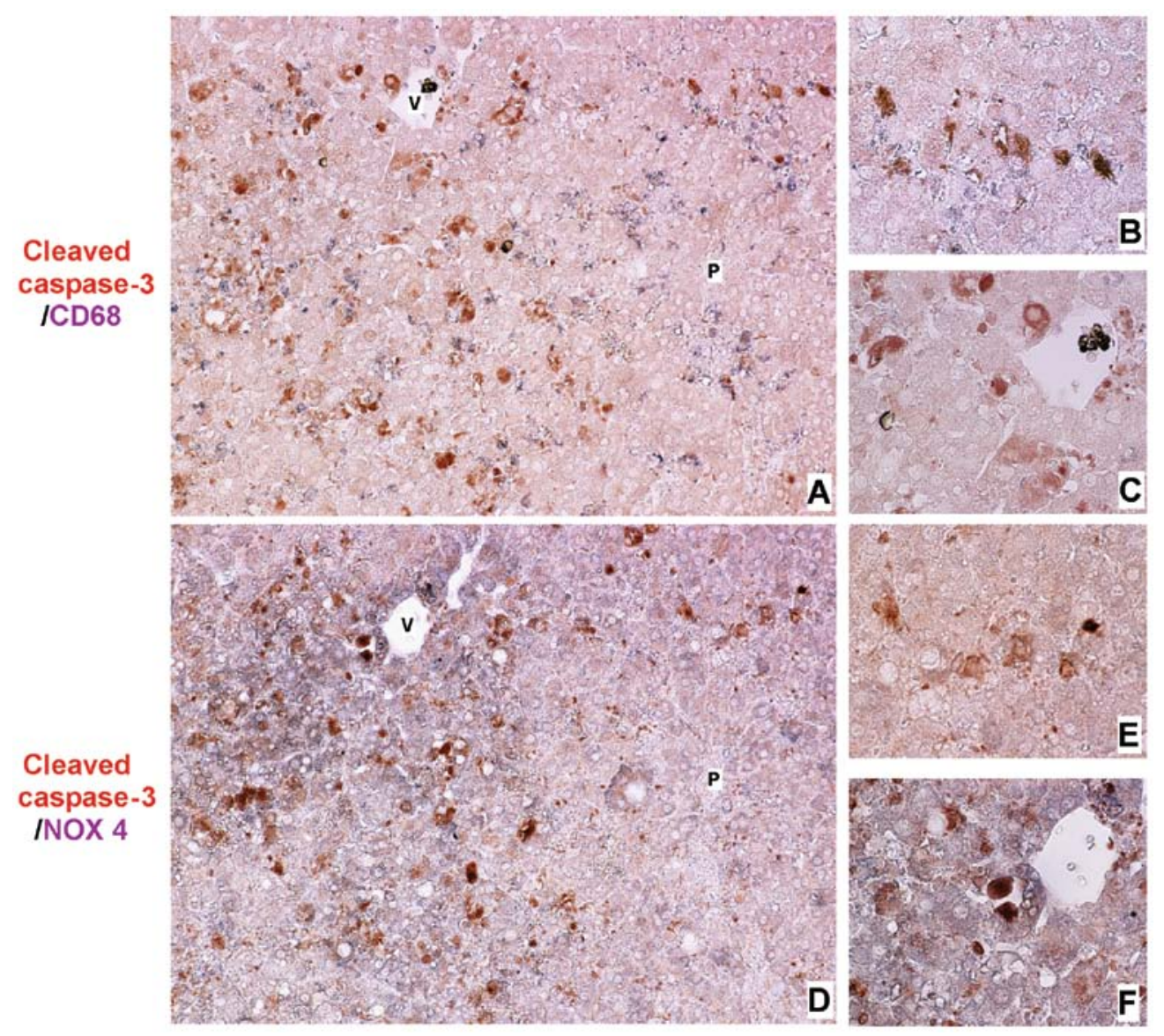

Figure 5. Simultaneous immunohistochemical detection of cleaved caspase-3 and CD68, and cleaved caspase-3 and nicotinamide adenine dinucleotide phosphate oxidase (NOX4) in livers of rats with acute liver failure (ALF). Immunohistochemical detection of cleaved caspase-3 (brown color) and CD68 (dark blue color) (A-C), and cleaved-caspase-3 (brown) and NOX4 (dark blue) (D-F) in livers of rats with ALF. The majority of apoptotic hepatocytes in zone 2 (B), and a few apoptotic hepatocytes in zone 3 (C) were localized adjacent to macrophages. A few apoptotic hepatocytes in zone 2 colocalized with NOX4 (E), and the majority of apoptotic hepatocytes in zone 3 were colocalized with NOX4 (F). Original magnification, x200 (A and D), x400 (B, C, E and F). P, portal vein; V, central vein. Zone 1, periportal zone; zone 2, intermediate zone; zone 3, pericentral zone.

that area (Fig. 2F). The number of infiltrated macrophages was most abundant in zone 2 , followed by zone 3 , and then zone 1 (Fig. 2G). The number of apoptotic hepatocytes was most abundant in zone 3 , followed by zone 2 then zone 1 (Fig. 3E). The difference in the distribution of macrophages and apoptotic cells strongly suggests that the activation of an apoptotic pathway in zone 3 may be promoted by factors other than macrophages.

Apoptosis is induced by the sequential activation of caspases. This activation can be triggered by the activation of death receptors located on cell membranes (extrinsic pathway), and by cellular stress, i.e., endoplasmic reticulum stress or oxidative stress (intrinsic pathway) (21). Among the possible enzymatic systems contributing to the oxidative stress of hepatocytes (xanthine oxidoreductase, cyclooxygenase, endothelial nitric oxide synthases, cytochrome P450 monoxygenases and NOX) (45), NOX is a major source of cellular ROS (22-24). ROS generation by NOX has been considered to occur only in phagocytes. Six homologues of phagocyte catalytic NOX (NOX2) have been isolated: NOX1, NOX3, NOX4, NOX5, DUOX1 and DUOX2 (46). NOX4 has been shown to have unique characteristics compared with other NOX homologues. NOX4 requires no cytosolic component for ROS-producing activity and produces large amounts of ROS constitutively $(47,48)$. NOX4 is expressed in fairly ubiquitous organs (49) and its expression levels are generally higher than those of other NOX homologues. NOX4 has therefore been suggested to be an inducible NOX (25). NOX4 has been shown to play a crucial role in the pathophysiology of cardiovascular diseases (23), diabetic complications (50) and fibrosis (51-53). In the liver, NOX4 is primarily expressed in hepatocytes, stellate cells and endothelial cells (54), and plays a pivotal role in the cellular senescence of hepatocytes (55), hepatitis C virusinduced ROS generation $(56,57)$ and liver fibrosis $(58-62)$.

Recently, NOX4 was found to be a target of HIF-1 $\alpha$, a master regulator of the cellular response to hypoxia (63), and therefore was suggested to be involved in cellular destruction in ALF. We hypothesized that intrahepatic microcirculatory disorder in the livers of rats with ALF may cause parenchymal hypoxia, and that the consequent activation of HIF1- $\alpha$ would upregulate NOX4, leading to the accumulation of oxidative stress and resultant hepatocyte apoptosis via an intrinsic pathway. In this study, zone 1 was almost normoxic and zone 3 was highly hypoxic (Fig. 4D). The hypoxic area was under impaired intrahepatic microcirculation (Fig. 4F) in the livers of rats with ALF. The results from immunohistochemistry for HIF1- $\alpha$ (Fig. 4G and H), 4-HNE (Fig. 4I and J) and NOX4 (Fig. 4K and L) suggested that their distributions were in accordance with those for pimoni- 
dazole. This indicates that oxidative stress may be induced by hypoxia-dependent NOX4 activation.

For a more precise evaluation of the localization of macrophages and NOX4-expressing cells in conjunction with that of apoptotic hepatocytes, we used simultaneous immunohistochemical staining. The majority of the apoptotic hepatocytes in zone 2 were localized adjacent to macrophages (Fig. 5B), but were not colocalized with NOX4-positive cells (Fig. 5E). The greatest portion of apoptotic hepatocytes in zone 3 was not localized adjacent to macrophages (Fig. 5C), but colocalized with NOX4-positive cells (Fig. 5F). These results indicated that hepatocyte apoptosis in zones 2 and 3 of our rat model of ALF was triggered mainly by activated macrophages and hypoxia, respectively.

To the best of our knowledge, ours is the first study to suggest that NOX4 upregulation may be involved in hepatocyte apoptosis during the progression of ALF. We recognize, however, that this study is based on histological observations only in one experimental animal model with no intervention. To date, NOX4-specific small-molecule inhibitors are not readily available $(64,65)$, and further studies involving genetic interventions of NOX4 in an ALF model are required.

In conclusion, the intravenous injection of TRITC-dextran clearly revealed intrahepatic microcirculation in FFPE sections, and demonstrated intrahepatic microcirculatory disorder in the livers of rats with ALF. This method enables the simultaneous examination of microcirculation and immunohistochemistry to be conducted on the same liver sections, and is useful for investigating correlations between sinusoidal blood flow and other physiological factors. Zonal differences in hepatocyte apoptosis were revealed. The data from the present study suggest that hypoxia in zone 3 and intrahepatic microcirculatory disorder followed by HIF1- $\alpha$ upregulation may induce NOX4 upregulation, thus resulting in cellular oxidative stress.

\section{Acknowledgements}

We appreciate the technical support from the Research Support Center, Graduate School of Medical Sciences, Kyushu University.

\section{References}

1. Lee WM, Squires RH, Nyberg SL, Doo E and Hoofnagle JH: Acute liver failure: Summary of a workshop. Hepatology 47: $1401-1415,2008$

2. Marudanayagam R, Shanmugam V, Gunson B, et al: Aetiology and outcome of acute liver failure. HPB (Oxford) 11: 429-434, 2009.

3. Oketani M, Ido A, Nakayama N, et al: Etiology and prognosis of fulminant hepatitis and late-onset hepatic failure in japan Summary of the annual nationwide survey between 2004 and 2009. Hepatol Res 43: 97-105, 2013.

4. Han DW: Intestinal endotoxemia as a pathogenetic mechanism in liver failure. World J Gastroenterol 8: 961-965, 2002.

5. Antoniades CG, Berry PA, Wendon JA and Vergani D: The importance of immune dysfunction in determining outcome in acute liver failure. J Hepatol 49: 845-861, 2008.

6. McCuskey RS, Urbaschek R, McCuskey PA and Urbaschek B: In vivo microscopic studies of the responses of the liver to endotoxin. Klin Wochenschr 60: 749-751, 1982.

7. Uhlmann S, Uhlmann D and Spiegel HU: Evaluation of hepatic microcirculation by in vivo microscopy. J Invest Surg 12: 179-193, 1999.

8. Klintman D, Schramm R, Menger MD and Thorlacius H: Leukocyte recruitment in hepatic injury: selectin-mediated leukocyte rolling is a prerequisite for CD18-dependent firm adhesion. J Hepatol 36: 53-59, 2002.
9. Eipel C, Kidess E, Abshagen K, et al: Antileukoproteinase protects against hepatic inflammation, but not apoptosis in the response of D-galactosamine-sensitized mice to lipopolysaccharide. Br J Pharmacol 151: 406-413, 2007.

10. Roller J, Laschke MW, Scheuer C and Menger MD: Heme oxygenase (HO)-1 protects from lipopolysaccharide (LPS)mediated liver injury by inhibition of hepatic leukocyte accumulation and improvement of microvascular perfusion. Langenbecks Arch Surg 395: 387-394, 2010.

11. Ito Y, Bethea NW, Abril ER and McCuskey RS: Early hepatic microvascular injury in response to acetaminophen toxicity. Microcirculation 10: 391-400, 2003.

12. Lawson JA, Fisher MA, Simmons CA, Farhood A and Jaeschke H: Parenchymal cell apoptosis as a signal for sinusoidal sequestration and transendothelial migration of neutrophils in murine models of endotoxin and Fas-antibody-induced liver injury. Hepatology 28: 761-767, 1998.

13. Fujiwara K, Ogata I, Ohta Y, et al: Intravascular coagulation in acute liver failure in rats and its treatment with antithrombin III. Gut 29: 1103-1108, 1988.

14. Yamada S, Ogata I, Hirata K, Mochida S, Tomiya T and Fujiwara K: Intravascular coagulation in the development of massive hepatic necrosis induced by Corynebacterium parvum and endotoxin in rats. Scand J Gastroenterol 24: 293-298, 1989.

15. Mochida S, Ohno A, Arai M, Tamatani T, Miyasaka M and Fujiwara K: Role of adhesion molecules in the development of massive hepatic necrosis in rats. Hepatology 23: 320-328, 1996.

16. Miyazaki M, Kato M, Tanaka M, et al: Antithrombin III injection via the portal vein suppresses liver damage. World J Gastroenterol 18: 1884-1891,2012.

17. Hirata K, Ogata I, Ohta Y and Fujiwara K: Hepatic sinusoidal cell destruction in the development of intravascular coagulation in acute liver failure of rats. J Pathol 158: 157-165, 1989.

18. Ding JW, Ning Q, Liu MF, et al: Fulminant hepatic failure in murine hepatitis virus strain 3 infection: tissue-specific expression of a novel fgl2 prothrombinase. J Virol 71: 9223-9230, 1997.

19. Mita A, Hashikura Y, Tagawa Y, Nakayama J, Kawakubo M and Miyagawa S: Expression of Fas ligand by hepatic macrophages in patients with fulminant hepatic failure. Am J Gastroenterol 100: 2551-2559, 2005.

20. Streetz K, Leifeld L, Grundmann D, et al: Tumor necrosis factor $\alpha$ in the pathogenesis of human and murine fulminant hepatic failure. Gastroenterology 119: 446-460, 2000.

21. Malhi $\mathrm{H}$ and Gores GJ: Cellular and molecular mechanisms of liver injury. Gastroenterology 134: 1641-1654, 2008.

22. Touyz RM, Briones AM, Sedeek M, Burger D and Montezano AC: NOX isoforms and reactive oxygen species in vascular health. Mol Interv 11: 27-35, 2011.

23. Chen F, Haigh S, Barman S and Fulton DJ: From form to function: the role of Nox4 in the cardiovascular system. Front Physiol 3: 412, 2012.

24. Katsuyama M: NOX/NADPH oxidase, the superoxide-generating enzyme: its transcriptional regulation and physiological roles. J Pharmacol Sci 114: 134-146, 2010.

25. Brown DI and Griendling KK: Nox proteins in signal transduction. Free Radic Biol Med 47: 1239-1253, 2009.

26. Arteel GE, Thurman RG, Yates JM and Raleigh JA: Evidence that hypoxia markers detect oxygen gradients in liver: pimonidazole and retrograde perfusion of rat liver. $\mathrm{Br} \mathrm{J}$ Cancer 72 : 889-895, 1995.

27. Arteel GE, Iimuro Y, Yin M, Raleigh JA and Thurman RG: Chronic enteral ethanol treatment causes hypoxia in rat liver tissue in vivo. Hepatology 25: 920-926, 1997.

28. Zhong Z, Arteel GE, Connor HD, et al: Binge drinking disturbs hepatic microcirculation after transplantation: prevention with free radical scavengers. J Pharmacol Exp Ther 290: 611-620, 1999.

29. Rosmorduc O, Wendum D, Corpechot C, et al: Hepatocellular hypoxia-induced vascular endothelial growth factor expression and angiogenesis in experimental biliary cirrhosis. Am J Pathol 155: 1065-1073, 1999.

30. Bardag-Gorce F, French BA, Li J, et al: The importance of cycling of blood alcohol levels in the pathogenesis of experimental alcoholic liver disease in rats. Gastroenterology 123: 325-335, 2002.

31. Corpechot C, Barbu V, Wendum D, et al: Hepatocyte growth factor and c-Met inhibition by hepatic cell hypoxia: a potential mechanism for liver regeneration failure in experimental cirrhosis. Am J Pathol 160: 613-620, 2002. 
32. Deng X, Luyendyk JP, Zou W, et al: Neutrophil interaction with the hemostatic system contributes to liver injury in rats cotreated with lipopolysaccharide and ranitidine. J Pharmacol Exp Ther 322: 852-861, 2007.

33. Chaudhuri S, McCullough SS, Hennings L, et al: Acetaminophen hepatotoxicity and HIF-1 $\alpha$ induction in acetaminophen toxicity in mice occurs without hypoxia. Toxicol Appl Pharmacol 252: 211-220, 2011.

34. Vollmar B, Wolf B, Siegmund S, Katsen AD and Menger MD: Lymph vessel expansion and function in the development of hepatic fibrosis and cirrhosis. Am J Pathol 151: 169-175, 1997.

35. Sun Z, Wang X, Deng X, et al: Beneficial effects of lexipafant a PAF antagonist on gut barrier dysfunction caused by intestinal ischemia and reperfusion in rats. Dig Surg 17: 57-65, 2000.

36. Takenaka K, Sakaida I, Yasunaga M and Okita K: Ultrastructural study of development of hepatic necrosis induced by TNF-alpha and D-galactosamine. Dig Dis Sci 43: 887-892, 1998.

37. Le Bail B, Bioulac-Sage P, Senuita R, Quinton A, Saric J and Balabaud C: Fine structure of hepatic sinusoids and sinusoidal cells in disease. J Electron Microsc Tech 14: 257-282, 1990.

38. Kotoh K, Kato M, Kohjima M, Nakamuta M and Enjoji M: A new treatment strategy for acute liver failure. World J Hepatol 2 : 395-400, 2010.

39. Levy GA, Liu M, Ding J, et al: Molecular and functional analysis of the human prothrombinase gene (HFGL2) and its role in viral hepatitis. Am J Pathol 156: 1217-1225, 2000

40. Marsden PA, Ning Q, Fung LS, et al: The Fgl2/fibroleukin prothrombinase contributes to immunologically mediated thrombosis in experimental and human viral hepatitis. J Clin Invest 112: 58-66, 2003.

41. Bantel H, Lugering A, Heidemann J, et al: Detection of apoptotic caspase activation in sera from patients with chronic HCV infection is associated with fibrotic liver injury. Hepatology 40 : $1078-1087,2004$

42. Rutherford AE, Hynan LS, Borges CBS, et al: Serum apoptosis markers in acute liver failure: a pilot study. Clin Gastroentero Hepatol 5: 1477-1483, 2007

43. Rutherford A, King LY, Hynan LS, et al: Development of an accurate index for predicting outcomes of patients with acute liver failure. Gastroenterology 143: 1237-1243, 2012

44. Malhi H, Gores GJ and Lemasters JJ: Apoptosis and necrosis in the liver: A tale of two deaths? Hepatology 43 (Suppl 1): S31-S44 2006.

45. De Minicis S, Bataller R and Brenner DA: NADPH oxidase in the liver: defensive, offensive, or fibrogenic? Gastroenterology 131: 272-275, 2006.

46. Bedard K and Krause KH: The NOX family of ROS-generating NADPH oxidases: physiology and pathophysiology. Physiol Rev 87: 245-313, 2007.

47. Martyn KD, Frederick LM, von Loehneysen K, Dinauer MC and Knaus UG: Functional analysis of Nox4 reveals unique characteristics compared to other NADPH oxidases. Cell Signal 18: 69-82, 2006.

48. Serrander L, Cartier L, Bedard K, et al: NOX4 activity is determined by mRNA levels and reveals a unique pattern of ROS generation. Biochem J 406: 105-114, 2007.

49. Krause KH: Tissue distribution and putative physiological function of NOX family NADPH oxidases. Jpn J Infect Dis 57 S28-S29, 2004

50. Meng D, Mei A, Liu J, et al: NADPH oxidase 4 mediates insulinstimulated HIF- $1 \alpha$ and VEGF expression, and angiogenesis in vitro. PLoS One 7: e48393, 2012.
51. Hecker L, Vittal R, Jones T, et al: NADPH oxidase-4 mediates myofibroblast activation and fibrogenic responses to lung injury. Nat Med 15: 1077-1081, 2009.

52. Barnes JL and Gorin Y: Myofibroblast differentiation during fibrosis: role of NAD(P)H oxidases. Kidney Int 79: 944-956, 2011.

53. Sedeek M, Callera G, Montezano A, et al: Critical role of Nox4-based NADPH oxidase in glucose-induced oxidative stress in the kidney: implications in type 2 diabetic nephropathy. Am J Physiol Renal Physiol 299: F1348-F1358, 2010.

54. Reinehr R, Becker S, Eberle A, Grether-Beck S and Haussinger D: Involvement of NADPH oxidase isoforms and Src family kinases in CD95-dependent hepatocyte apoptosis. J Biol Chem 280 27179-27194, 2005.

55. Senturk S, Mumcuoglu M, Gursoy-Yuzugullu O, Cingoz B Akcali KC and Ozturk M:. Transforming growth factor-beta induces senescence in hepatocellular carcinoma cells and inhibits tumor growth. Hepatology 52: 966-974, 2010.

56. Boudreau HE, Emerson SU, Korzeniowska A, Jendrysik MA and Leto TL: Hepatitis C virus (HCV) proteins induce NADPH oxidase 4 expression in a transforming growth factor betadependent manner: a new contributor to HCV-induced oxidative stress. J Virol 83: 12934-12946, 2009.

57. de Mochel NS, Seronello S, Wang SH, et al: Hepatocyte NAD(P) $\mathrm{H}$ oxidases as an endogenous source of reactive oxygen species during hepatitis $C$ virus infection. Hepatology 52: 47-59, 2010.

58. Carmona-Cuenca I, Roncero C, Sancho P, et al: Upregulation of the NADPH oxidase NOX4 by TGF-beta in hepatocytes is required for its pro-apoptotic activity. J Hepatol 49: 965-976, 2008.

59. Caja L, Sancho P, Bertran E, Iglesias-Serret D, Gil J and Fabregat I: Overactivation of the MEK/ERK pathway in liver tumor cells confers resistance to TGF- $\{$ beta\}-induced cell death through impairing up-regulation of the NADPH oxidase NOX4. Cancer Res 69: 7595-7602, 2009.

60. Carmona-Cuenca I, Herrera B, Ventura JJ, Roncero C, Fernandez $\mathrm{M}$ and Fabregat I: EGF blocks NADPH oxidase activation by TGF-beta in fetal rat hepatocytes, impairing oxidative stress, and cell death. J Cell Physiol 207: 322-330, 2006

61. Sancho P, Bertran E, Caja L, Carmona-Cuenca I, Murillo MM and Fabregat I: The inhibition of the epidermal growth factor (EGF) pathway enhances TGF-beta-induced apoptosis in rat hepatoma cells through inducing oxidative stress coincident with a change in the expression pattern of the NADPH oxidases (NOX) isoforms. Biochim Biophys Acta 1793: 253-263, 2009.

62. Sancho P, Mainez J, Crosas-Molist E, et al: NADPH oxidase NOX4 mediates stellate cell activation and hepatocyte cell death during liver fibrosis development. PLoS One 7: e45285, 2012.

63. Diebold I, Petry A, Hess J and Görlach A: The NADPH oxidase subunit NOX4 is a new target gene of the hypoxia-inducible factor-1. Mol Biol Cell 21: 2087-2096, 2010.

64. Borbely G, Szabadkai I, Horvath Z, et al: Small-molecule inhibitors of NADPH oxidase 4. J Med Chem 53: 6758-6762, 2010.

65. Cifuentes-Pagano E, Csanyi G and Pagano PJ: NADPH oxidase inhibitors: a decade of discovery from Nox2ds to HTS. Cell Mol Life Sci 69: 2315-2325, 2012.

66. Henriques U: Histological technique in routine histopathology. An opinion. Pathol Res Pract 171: 417-422, 1981. 